\title{
Human Psychopharmacology:
}

Clinical \& Experimental

\section{Modelling PTSD Diagnosis Using Sleep, Memory and Adrenergic Metabolites: An Exploratory Machine-Learning Study.}

\begin{tabular}{|c|c|}
\hline Journal: & Human Psychopharmacology: Clinical and Experimental \\
\hline Manuscript ID & HUP-18-0010.R2 \\
\hline Wiley - Manuscript type: & Research Article \\
\hline $\begin{array}{r}\text { Date Submitted by the } \\
\text { Author: }\end{array}$ & $\mathrm{n} / \mathrm{a}$ \\
\hline Complete List of Authors: & $\begin{array}{l}\text { Breen, Michael; Icahn School of Medicine at Mount Sinai Department of } \\
\text { Genetics and Genomic Sciences } \\
\text { Thomas, Kevin; University of Cape Town, Department of Psychology } \\
\text { Baldwin, David; University of Southampton, } \\
\text { Lipinska, Gosia; University of Cape Town, Department of Psychology }\end{array}$ \\
\hline Keyword: & PTSD, sleep, memory, diagnosis, machine learning, metabolites \\
\hline
\end{tabular}

\section{SCHOLARONE \\ Manuscripts}


Role of Sleep, Memory and Adrenergic Metabolites in PTSD

Title: Modelling PTSD Diagnosis Using Sleep, Memory and Adrenergic Metabolites: An Exploratory Machine-Learning Study.

Michael S. Breen ${ }^{a, b}$, Kevin G. F. Thomas ${ }^{c}$, David S. Baldwin ${ }^{d, e}$, Gosia Lipinskac

aDepartment of Psychiatry, 'bepartment of Genetics and Genomic Sciences, Icahn School of Medicine at Mount Sinai, New York, New York, USA.; ' Department of Psychology, University of Cape Town, Cape Town, South Africa; ${ }^{d}$ Clinical and Experimental Sciences, University of Southampton, Southampton, UK; eDepartment of Psychiatry and Mental Health, University of Cape Town, Cape Town, South Africa.

\section{Corresponding Author:}

Gosia Lipinska

Department of Psychology

University of Cape Town, Private Bag

Rondebosch 7701, South Africa

Tel: +27-21-650 3435

gosia.lipinska@uct.ac.za

\section{Conflict of Interest}

None

\section{Funding Declaration}

This work was supported by the A.W. Mellon Foundation and the National Research Foundation. These funders provided individual financial support to GL - they had no role in the study design; collection, analysis and interpretation of the data; writing of the report; or in the decision to submit the article for publication.

Keywords: PTSD, sleep, memory, diagnosis, machine learning, metabolites 


\begin{abstract}
Objective. Features of posttraumatic stress disorder (PTSD) typically include sleep disturbances, impaired declarative memory and hyperarousal. This study evaluated whether these combined features may accurately delineate pathophysiological changes associated with PTSD.
\end{abstract}

Method. We recruited a cohort of PTSD-diagnosed individuals $(N=20)$, trauma survivors without PTSD (TE; $N=20)$ and healthy controls $(\mathrm{HC} ; \mathrm{N}=20)$. Analyses of between-group differences and support vector machine (SVM)-learning were applied to participant features.

Results. Analyses of between-group differences replicated previous findings, indicating that PTSD diagnosed individuals self-reported poorer sleep quality, objectively demonstrated less sleep depth, and evidenced declarative memory deficits in comparison to $\mathrm{HC}$. Integrative SVM-learning distinguished HC from trauma participants with $80 \%$ accuracy using a combination of five features, including subjective and objective sleep, neutral declarative memory and metabolite variables. PTSD and TE participants could be distinguished with $70 \%$ accuracy using a combination of subjective and objective sleep variables, but not by metabolite or declarative memory variables.

Conclusion. From among a broad range of sleep, cognitive and biochemical variables, sleep characteristics were the primary features that could differentiate those with PTSD from those without. Our exploratory SVM-learning analysis establishes a framework for future sleep- and memory-based PTSD investigations that could drive improvements in diagnostic accuracy and treatment. 
Role of Sleep, Memory and Adrenergic Metabolites in PTSD

\section{BACKGROUND}

Sleep disturbances and memory deficits are prominent features of posttraumatic stress disorder (PTSD), and are also strongly implicated in PTSD susceptibility and symptom development (Brewin, Kleiner, Vasterling, \& Field, 2007; Germain, 2013). Although these disturbances and deficits are sometimes considered secondary symptoms of the disorder, disturbing dreams, insomnia and poor memory are reported by most diagnosed individuals (Agorastos, Kellner, Baker, \& Otte, 2014; Pace-Schott, Germain, \& Milad, 2015). Furthermore, recent evidence suggests that these sleep and memory-related alterations may be meaningfully associated, because of the role that sleep plays in memory consolidation (Feld \& Diekelmann, 2015; Goerke, Muller, \& Cohrs, 2017; Lipinska, Timol, Kaminer, \& Thomas, 2014). A better understanding of the role of trauma-induced sleep and memory disturbances in PTSD development may help drive improved diagnostic and treatment approaches.

Data derived from structured clinical interviews and self-report symptom checklists and questionnaires suggest that PTSDdiagnosed individuals consistently experience numerous forms of sleep disruption. These include difficulties in falling asleep, frequent awakenings from sleep, shorter sleep duration, restless sleep, 
Role of Sleep, Memory and Adrenergic Metabolites in PTSD

daytime fatigue and nightmares (Buysse et al., 2008; Giosan et al., 2015; Werner, Griffin, \& Galovski, 2016).

However, investigations using polysomnography (PSG) have produced inconsistent results regarding the nature and magnitude of sleep disturbances in PTSD. Some studies have found decreased rapid eye movement (REM) duration during sleep (Hefez, Metz, \& Lavie, 1987; Lavie, Hefez, Halperin, \& Enoch, 1979; Lipinska et al., 2014; Mikulincer, Glaubman, Wasserman, Porat, \& Birger, 1989), whereas others have found either normal or prolonged REM durations (Dow, Kelsoe, \& Gillin, 1996; Engdahl, Eberly, Hurwitz, Mahowald, \& Blake, 2000; Glaubman, Miculincer, Porat, Wasserman, \& Birger, 1990; Mellman, Kulick-Bell, Ashlock, \& Nolan, 1995). Similarly, abnormalities in slow-wave sleep (SWS) are found inconsistently, with some studies reporting decreased SWS in PTSD-diagnosed individuals relative to controls (Fuller, Waters, \& Scott, 1994; Glaubman et al., 1990; Habukawa, Uchimura, Maeda, Kotorii, \& Maeda, 2007; Mikulincer et al., 1989; Yetkin, Aydin, \& Ozgen, 2010), whereas others report no between-group differences (Dow et al., 1996; Hurwitz, Mahowald, Kuskowski, \& Engdahl, 1998; Mellman, Kulick-Bell, et al., 1995; Ross et al., 1994; Woodward, Murburg, \& Bliwise, 2000). Meta-analyses attribute these inconsistencies to between-study differences in methodology, as well as variability in controlling for moderating variables such as age, gender, trauma type, and psychiatric comorbidities that may 
Role of Sleep, Memory and Adrenergic Metabolites in PTSD

influence the nature and extent of sleep disruption (Kobayashi, Boarts, \& Delahanty, 2007).

Inconsistences may also be related to the lack of comprehensive symptom descriptions. For instance, the frequency and magnitude of hyperarousal symptoms are rarely described, even though these have been linked with disturbed sleep (Kobayashi, Lavela, \& Mellman, 2014; Mellman, Kumar, Kulick-Bell, Kumar, \& Nolan, 1995; van Liempt et al., 2013). In these studies, hyperarousal symptoms have been indexed using self-report measures, psychophysiological measures (heart rate variability) and a number of biomarkers (levels of cortisol, adrenocorticotropic hormone and noradrenaline derived from plasma or urine metabolites). Measures of noradrenergic functioning are particularly useful in characterising the relationship between sleep disturbance and hyperarousal in PTSD, because noradrenaline is activated during sympathetic activation of the autonomic nervous system under conditions of perceived or actual threat. Furthermore, Mellman et al. (1995) found that PTSDdiagnosed participants, in comparison with healthy controls, had elevated night-time noradrenergic activity, and that the extent of this activation was correlated with the degree of sleep disturbance. However, the lack of consistent objective sleep findings indicates a need for improved biomarker approaches that might help differentiate reliably between those with and without a diagnosis of PTSD. 
Role of Sleep, Memory and Adrenergic Metabolites in PTSD

A large body of research applying memory measures (including listlearning, narrative recall tasks, and paired associated learning) to the study of PTSD has demonstrated prominent memory deficits, associated with small-to-moderate effect sizes, in affected individuals (Brewin et al., 2007; Johnsen \& Asbjornsen, 2008). Such deficits are strongly related to reduced hippocampal volume in PTSD-diagnosed individuals (Bremner et al., 1995; Vythilingam et al., 2005). Recent research investigating the importance of healthy sleep (specifically, intact sequences of, and transitions between, early-night SWS and late-night REM sleep) for the processing and consolidation of memories encoded during waking has indicated that, in PTSD, disturbed sleep and poor performance on recall and recognition tasks may be closely related. Independent studies have demonstrated that either disrupted REM sleep (Lipinska et al., 2014) or fragmented early night SWS-rich sleep (van Liempt, Vermetten, Lentjes, Arends, \& Westenberg, 2011) is associated with poor performance on post-sleep declarative memory tests. Irrespective of the kind of disruption in sleep architecture, these studies highlight that sleep and memory disruptions in PTSD are not isolated symptoms, but instead are meaningfully related, with potential mechanistic interaction. However, no study has investigated the potential utility of this constellation of features in exploring the correlates of PTSD diagnosis. Investigation of these features may 
Role of Sleep, Memory and Adrenergic Metabolites in PTSD

augment our understanding of current diagnostic criteria and provide deeper insight into the structure of post-traumatic symptoms.

The present study assessed the utility of combining 74 different features including subjectively and objectively measured sleep disturbance, declarative memory deficits and changes in biological markers of hyperarousal (noradrenergic and adrenergic metabolites) across those diagnosed with PTSD, those exposed to trauma and healthy controls. Our objective was to identify the minimum combination of features with the highest diagnostic accuracy to characterise PTSD. We conducted this exploration using supervised multivariate classification methods to integrate and identify a combination of features that may be capable of accurate PTSD classification.

\section{METHODS}

This study, which was part of a larger study investigating relations between cognition, affect, and sleep in PTSD-diagnosed individuals (Lipinska \& Thomas, 2017), featured a cross-sectional quasiexperimental design. 
Role of Sleep, Memory and Adrenergic Metabolites in PTSD

\subsection{Participants}

Sixty women were enrolled into the study and assigned to three groups ( $N=20$ each): PTSD, trauma survivors without PTSD (TE), and healthy controls $(\mathrm{HC})$. We opted to recruit participants in such a way as to be able to detect any significant results based on medium effect sizes, using a conservative estimate of $f=0.33$ from a previous study using a similar cohort (effect sizes range: $f=0.33$ to $f=0.73$; Lipinska et al., 2014). A power analysis revealed that to achieve power of 0.8 with $\alpha$ set at 0.05 , we needed to recruit at least 60 participants.

All participants in the PTSD and TE groups were adults sexual assault survivors who had experienced a single sexual assault, which was considered the index trauma. Participants were recruited through a local non-profit organisation that provides a counselling service to sexual assault survivors, and through advertisements placed in local newspapers. The three groups were aggregatematched on age (for the entire sample, $25.3 \pm 4.1$ years), general intellectual functioning (for the entire sample, Wechsler $I Q=82.3 \pm$ 14.2), level of income (for the entire sample, $4706.0 \pm 2828.0$ ZAR), and smoking habits $(13 \%$ of all participants smoked cigarettes regularly). The sample included seven participants with asymptomatic HIV. The PTSD and TE groups were aggregate matched on time since trauma (overall, $1.3 \pm 1.0$ years) (Table 1 ). 
All participants had no history of childhood sexual trauma and of alcohol or illicit substance abuse, and were free of psychoactive medication. Full ethical approval was obtained from the relevant bodies at the hosting institution, and all participants provided written informed consent.

A trained researcher used the Clinician-Administered PTSD Scale (CAPS; Blake et al., 1995) to measure the presence and severity of PTSD symptoms. We used a moderate scoring rule which stipulated that a CAPS score of at least 45 must be reached for a diagnosis of PTSD to be made, and that there must be the appropriate Diagnostic and Statistical Manual, Fourth Edition, Text Revision (DSM-IV TR) distribution of symptoms across clusters. PTSD diagnosis was confirmed using the Mini International Neuropsychiatric Inventory (MINI; Sheehan et al., 1998). Participants had to meet both CAPS and MINI PTSD diagnosis criteria to remain eligible for inclusion in the PTSD group. Presence and severity of depression was rated using the Beck Depression Inventory (BDI-II; Beck, Steer, \& Brown, 1996). To provide an estimate of general intellectual functioning, participants completed the Performance subtests of the Wechsler Abbreviated Scale of Intelligence (WASI; D. Wechsler, 1999). Potential participants were excluded from the PTSD and TE groups if they had a current primary psychiatric diagnosis that was not secondary to trauma and were excluded from the $\mathrm{HC}$ group if they displayed any psychiatric diagnosis. Eligible participants were 
Role of Sleep, Memory and Adrenergic Metabolites in PTSD

scheduled for two night sessions and one day session, and were required to refrain from vigorous exercise, alcohol, nicotine, and caffeinated drinks for $12 \mathrm{~h}$ prior to testing.

\subsection{Sleep-wake assessment and memory performance}

A blocked randomisation approach assigned an equal number of participants from each group to either two consecutive night sessions followed by a day session, or a day session followed by two consecutive night sessions. The day and the night sessions were separated by at least $48 \mathrm{~h}$ and occurred within a week of each other.

For the night sessions, participants arrived at the sleep laboratory approximately $2 \mathrm{~h}$ before their normal bedtime. The first night (N1) was an adaptation night to familiarise them with the environment and with the equipment used to monitor their sleep. Prior to bedtime during N1, participants completed the Pittsburgh Sleep Quality Index (PSQI; Buysse, Reynolds, Monk, Berman, \& Kupfer, 1989) to characterise subjective sleep quality in their home environment. Prior to bedtime on the second night (N2), participants completed the Logical Memory (LM) and Word List (WL) subtests of the Wechsler Memory Scale-Third Edition (WMS-III; D. Wechsler, 1997) and the Story Memory (SM) subtest of the Wide Range Assessment of Memory and Learning (WRAML; Sheslow \& Adams, 2003). Administration of these tests followed standardised procedures. That 
is to say, an immediate recall trial followed initial presentation of each stimulus set (LM and SM: a one-paragraph story in each case; WL: a list of 12 semantically unrelated words), and an uncued delayed recall trial followed some time after that. The only exception to the standard administration procedures was that the delayed recall trial followed a period of sleep, rather than the usual 25-35 mins of filled activity. After completing that set of cognitive tests, participants prepared for bedtime and were attached to a 16-channel Nihon Kohden NeuroFax EEG9000 electroencephalograph (EEG) adapted for sleep research.

Upon waking after an 8-hour sleep opportunity, an adapted version of the PSQI (Laboratory PSQI; Supplementary Appendix A) assessed subjective laboratory sleep during the night, and delayed free and cued recall trials of the three memory tasks (LM, WL, SM) were administered. Both the PSQI and Laboratory PSQI were scored, following convention, to calculate a Global Score capturing overall sleep quality over the reporting period (Buysse et al., 1989).

For the day session, participants arrived at the laboratory at approximately $08 \mathrm{~h} 00$ and completed parallel versions of the LM, WL, and SM immediate recall tasks. After $8 \mathrm{~h}$ of waking activity, they returned to the lab so that delayed free tasks could be administered. Participants were instructed to carry out their ordinary daily activities during the $8 \mathrm{~h}$ wake period, but not to nap. To estimate declarative 
Role of Sleep, Memory and Adrenergic Metabolites in PTSD

memory performance, immediate recall, delayed recall, and retention (i.e., comparison of immediate with delayed recall, expressed as a percentage) scores were derived from each of the LM, WL, and SM tasks. Three composite scores were also calculated averaging immediate recall, delayed recall, and retention Z-scores across tasks. Retention was the primary variable of interest.

\subsection{PSG acquisition, analysis and data pre-processing}

To ensure reliable PSG recording, we applied a combination referential and bipolar montage with the bipolar derivations being F3C3, C3-P3, P3-O1, and F4-C4, C4-P4, P4-O2, and the referential derivations being F3-A2, C3-A2, O3-A2, and F4-A1, C4-A1, O4-A1. Eye movements, muscle tonus, and heart rate were recorded on two electrooculography (EOG) channels, and on one electromyography (EMG) and one electrocardiography (ECG) channel, respectively. Standardised filters for recording sleep were employed for the EEG and EOG $(0.5-35 \mathrm{~Hz})$, EMG $(10-70 \mathrm{~Hz})$ and ECG $(1-70 \mathrm{~Hz})$ leads to ensure integrity of the signal. The ground electrode was placed on the middle of the forehead.

PSG records were analysed according to Rechtschaffen and Kales (1968) criteria, for twelve sleep-related outcome variables: sleep latency; sleep efficiency; number of awakenings (defined as a period of wakefulness longer than $1.5 \mathrm{~min}$ after sleep onset); number of 
Role of Sleep, Memory and Adrenergic Metabolites in PTSD

spontaneous arousals (defined as a period of abrupt PSG shift during the night, usually an increase in PSG frequency lasting at least 3s but less than $1.5 \mathrm{~min}$ ); number of minutes spent awake after sleep onset (WASO); non-rapid eye movement (NREM) stage 1 percentage (NREM 1\%); NREM stage 2 percentage (NREM 2\%); SWS percentage (SWS\%); REM percentage (REM\%); REM latency; REM spontaneous arousals (REM arousals); and REM arousals leading to waking or NREM 1 sleep (REM NREM1/W). G.L. scored all sleep records while blind to group assignment.

\subsection{Measurement of noradrenergic and adrenergic metabolites}

Participants were asked to micturate into containers holding $10 \mathrm{ml} 6 \mathrm{M}$ $\mathrm{HCl}$ preservative during three periods: (1) 08h00-16h00, (2) 16h00$24 \mathrm{~h} 00$, and (3) $24 \mathrm{~h} 00-08 \mathrm{~h} 00$. The first two collection periods occurred within the day session, whereas the third occurred during the N2 session. During each collection period, participants voided into a receptacle and transferred their urine to a convenient and appropriately marked small cooler box or kept directly at $4^{\circ} \mathrm{C}$. Eventually, each bottle was transferred to the local National Health Laboratory Service where volumes for the three collections were measured and aliquots obtained. The samples were then frozen at $80^{\circ}$ for assay. The samples were then analysed using gas chromatography (GC) and mass spectrometry (MS). An Agilent Technology 7890A gas chromatographer and 5975C mass 
Role of Sleep, Memory and Adrenergic Metabolites in PTSD

spectrometer were used for analysis. Samples were analysed according to standard GC-MS procedures described elsewhere (Burtis, Ashwood, \& Bruns, 2012; Naccarato, Gionfriddo, Sindona, \& Tagarelli, 2014). Such analysis of urinary-derived normetadrenaline and metadrenaline metabolites is considered a robust, valid, and reliable method to estimate noradrenergic and adrenergic activity respectively. Furthermore, this analytic procedure provides adequate protection against interference from drugs, drug metabolites, exercise, and stress (Peaston \& Weinkove, 2004)

\subsection{Statistical Analyses}

Prior to SVM analysis, we conducted a number of conventional analyses to examine between-group differences with respect to sleep, memory and metabolite measures. Data related to these variables were assessed for normality with the Shapiro-Wilk test, and then either a one-way analysis of variance (ANOVA) or a KruskalWallis ANOVA with Tukey HSD post hoc correction was implemented, respectively. Tukey's method was used to create confidence intervals for all pairwise differences between-group level means while controlling for $95 \%$ family error rate. Models were adjusted for age, nicotine, and HIV status to control for potential confounding effects on PSG. Correlations among clinical assessment measures (CAPS and PSQI) across PTSD and TE groups were estimated using Spearman coefficient. Significant 
Role of Sleep, Memory and Adrenergic Metabolites in PTSD

correlations were inspected for outlying data points, which were removed accordingly.

\subsubsection{Machine-learning analyses}

Our dataset comprised 74 different features of PTSD, including subjective sleep, objective sleep, and memory and metabolite variables, across three groups (PTSD, TE and HC). Our objective was to identify the minimum combination of features with the highest diagnostic accuracy. Data pre-processing handled missing data using the kNNimputation package in R (Torgo, 2010), applying a non-parametric nearest neighbour method, akin to previous reports (Galatzer-Levy, Karstoft, Statnikov, \& Shalev, 2014). ${ }^{1}$ Categorical variables were then treatment coded (e.g. 0,1) and continuous variables were Z-transformed in order to normalise the range and variance of all data measured by disparate technologies and clinical nosology. All variables were then subjected to BRB-Array Tools (Simon et al., 2007) supervised classification methods to construct PTSD classifiers. Two separate models were specified: (1) HC vs. trauma-exposed participants (PTSD and TE), and (2) PTSD vs. TE participants. Each model consisted of three steps. First, to ensure a fair comparison, all variables were subjected to classifier construction. This heuristic approach was used to cast a wide net to

\footnotetext{
${ }^{1} \mathrm{~A}$ total of 31 missing data points across 20 different measures were imputed. This represents $\sim 0.6 \%$ of the total data used in our analyses
} 
catch all potentially informative measurements, while false positives would be pared off by subsequent optimisation and cross-validation steps. $^{2}$ Second, classifiers composed of different numbers of measurements were constructed by recursive feature elimination (RFE), which provided feature selection, model fitting, and performance evaluation through identifying the optimal number of features with maximum predictive accuracy. ${ }^{3}$ RFE is a feature selection method that fits a model and removes the weakest feature (or features) until the specified number of features is reached. Features are ranked by the model's feature importance attributes, and by recursively eliminating a small number of features per loop, RFE attempts to eliminate dependencies and collinearity that may exist in the model.Third, the ability of RFE to predict group outcome was assessed by support vector machines and compared with four different multivariate classification methods (that is, diagonal linear discriminant analysis, nearest centroid, first-nearest neighbours and three-nearest neighbours) in a nested leave-one-out cross-validation approach. Three notable advantages of this approach, include: i) its

\footnotetext{
${ }^{2}$ In cross-validation, data are broken up into $K$ partitions and then, $K$ times in turn, one partition is selected for testing while the the remaining ones are used for training. We used a nested crossvalidation, which uses an outer k-fold cross-validation loop to split the data into training and test folds, while an inner loop is used to select the model via k-fold cross-validation on the training fold only. After model selection, the test fold is then used to evaluate the model performance. Supplemental Figure 1 is a graphic depiction of these points.

${ }^{3} \mathrm{RFE}$ is a feature selection method that fits a model and removes the weakest feature (or features) until the specified number of features is reached. Features are ranked by the model's feature importance attributes, and by recursively eliminating a small number of features per loop, RFE attempts to eliminate dependencies and collinearity that may exist in the model.
} 
Role of Sleep, Memory and Adrenergic Metabolites in PTSD

use of maximum-margin hyper-planes in order to maximise the SVMs ability to predict the correct classification of previously unseen samples using small incremental changes; ii) the use of nested leave-one-out cross validation; and iii) the use of RFE to identify a unique set of features with maximum predictive value. The comparison between and amongst these four machine learning methods have been reviewed elsewhere (Simon et al., 2007). A detailed schematic of the current approach is summarized in Supplemental Figure 1.

\section{RESULTS}

\subsection{Clinical characteristics of PTSD and TE participants}

Table 2 shows the correlations between PSQI Global Score and CAPS Total Score with other clinical measures. Participants' BDI-II Score, PSQI Global Score and CAPS total score showed medium to strong positive correlations with each other, indicating that higher severities of depression, sleep disruption and PTSD were all associated with one another.

\subsection{PSG and declarative memory analysis}

Between-group analyses on PSG data from N2 showed that, compared to HC participants, PTSD and TE participants displayed 
Role of Sleep, Memory and Adrenergic Metabolites in PTSD

prolonged NREM $1 \% \quad(p=0.04$ and $p=0.03$, respectively $)$, and decreased SWS $\% \quad(p=0.01$ and $p=0.02$, respectively; see Supplementary Table 1)

Declarative memory performance was measured during the day and N2 sessions (Supplementary Table 2). Regarding data from the night sessions, PTSD participants performed significantly more poorly than $\mathrm{HC}$ on a) $\mathrm{LM}$ delayed recall, b) $\mathrm{LM}$ retention, c) $\mathrm{WL}$ delayed recall, $d$ ) delayed recall composite, and e) retention composite $(p=.05, p=.04, p=.01, p=.02$, and $p=.001$ respectively). TE participants performed more poorly than $\mathrm{HC}$ on a) LM delayed recall, $b$ ) retention composite and $c$ ) WL delayed recall ( $p=.01, p=.001$, and $p=.02$ respectively). Analyses of data from the day session detected no significant between-group differences.

\subsection{Noradrenergic and adrenergic metabolite analysis}

No significant differences were observed in metabolite levels either between groups or at any of the specified collection times (Supplementary Table 3). Additionally, no metabolite measurement was significantly associated with PSQI Global Score or with the CAPS Total Score. 
Role of Sleep, Memory and Adrenergic Metabolites in PTSD

\subsection{Exploring PTSD classification with machine-learning}

Using SVM-learning two separate models were specified to distinguish between: 1) HC vs. trauma groups (PTSD and TE); and 2) PTSD vs. TE. We identified five features able to discriminate $\mathrm{HC}$ from PTSD and TE participants with $80 \%$ accuracy (Figure 1A-C and Supplementary Table 4A and 4B). The composition of this classifier was populated with one memory feature (WL delayed recall, night session), three sleep features (PSQI, SWS\% N2, awakenings N2), and one biological marker (metanephrine 16h0024h00) (Figure 1B and Supplementary Table 4A). The second model identified five features able to discriminate PTSD from TE participants with $70 \%$ accuracy using SVM classification (Figure 1DF and Supplementary Table 4B). The composition of this classifier was populated with five sleep features (PSQI, subjective sleep N2, normal bedtime, arousals N2, and awakenings N2) (Figure 1E).

\section{DISCUSSION}

We set out to investigate relationships between sleep, memory, and noradrenergic and adrenergic metabolites in PTSD using conventional parametric/non-parametric analyses and exploratory supervised multivariate classification. Analyses of data from a primary cohort of 20 PTSD, 20 trauma-exposed, and 20 healthy control participants controlled for age, smoking and HIV status, as 
Role of Sleep, Memory and Adrenergic Metabolites in PTSD

these variables have the potential to confound outcomes related to sleep and memory (Lo, Groeger, Cheng, Dijk, \& Chee, 2016; Watkins \& Treisman, 2015). Initial between-group and correlational analyses replicated previous findings, indicating that PTSD diagnosed individuals self-reported poorer sleep quality, objectively demonstrated less sleep depth and evidenced deficits in neutral declarative memory in comparison to healthy controls. The novelty of the current paper, however, lies in findings from exploratory SVMlearning analysis. That analysis suggests that a combination of sleep (measured both objectively and subjectively), metabolite and neutral declarative memory variables could differentiate those with trauma exposure from healthy controls with $80 \%$ accuracy. Furthermore, PTSD and TE participants could be differentiated from one another, with $70 \%$ accuracy, by a combination of subjective and objective sleep variables (but not metabolite or neutral declarative memory variables). Thus, from among a broad range of clinical features, sleep characteristics were the primary features that differentiated those with PTSD from those without.

Our findings are consistent with previous reports suggesting sleep disruption in PTSD is central to the disorder, rather than merely a secondary symptom (Agorastos et al., 2014; Pigeon \& Gallegos, 2015; Spoormaker \& Montgomery, 2008). Indeed, some studies indicate that sleep disruption, specifically that related to REM fragmentation, is predictive of development of PTSD following 
trauma (Mellman, Bustamante, Fins, Pigeon, \& Nolan, 2002). In other words, there are strong suggestions that sleep quality is a key factor in the transition between trauma experience and resilience or development of psychopathology. Furthermore, several randomised control trials have demonstrated that both psychotherapeutic and pharmacological treatments aimed specifically at sleep disruption in PTSD are effective not only in treating sleep disturbance, but also ameliorate other symptoms of the disorder (Davis \& Wright, 2007; Germain et al., 2012; Raskind et al., 2013; Talbot et al., 2014). Together, these strands of the literature suggest that sleep disruption is not an isolated symptom of PTSD, and that it may, in fact, be mechanistically associated with other cognitive, emotional, and endocrine functions within the disorder.

\subsection{Features differentiating PTSD, TE and HC participants}

Five features (three related to subjective measures of sleep quality, and two related to PSG measures) distinguished participants as belonging to either the PTSD or the TE group (Figure 1 D-F): subjective laboratory sleep quality, subjective home sleep quality, normal bedtime, number of spontaneous arousals during laboratory sleep, and number of awakenings during laboratory sleep. Regarding the three subjective sleep features, better subjective laboratory sleep quality, poorer subjective home sleep, and earlier bedtime were associated with a PTSD diagnosis rather than with 
Role of Sleep, Memory and Adrenergic Metabolites in PTSD

trauma exposure alone. Although these classifiers contrast with respect to subjective sleep quality between the laboratory and home environment, previous studies have found that PTSD-diagnosed individuals self-report better sleep quality in the laboratory than in the home environment (Hurwitz et al., 1998; Kobayashi, Huntley, Lavela, \& Mellman, 2012; Lipinska \& Thomas, 2017; Spoormaker \& Montgomery, 2008). Furthermore, although at first glance it might be surprising that PTSD-diagnosed individuals tended to have earlier bed-times, we speculate that the nature of their home environments might explain this. Specifically, those carrying the diagnosis may go to be earlier in an attempt to avoid amplified feelings of nighttime insecurity and vulnerability associated with the objective reality of danger present in South African townships.

Regarding PSG measures, increased spontaneous arousals and awakenings were also associated with a PTSD diagnosis, rather than with trauma exposure alone. This finding suggests that despite fairquality self-reported sleep in the laboratory, PTSD-diagnosed individuals objectively have more fragmented sleep than TE participants. Several recent studies have demonstrated that fragmentation of sleep is an important characteristic of sleep disruption in PTSD (Mellman et al., 2002; Mellman, Kobayashi, Lavela, Wilson, \& Hall Brown, 2014; van Liempt et al., 2011).

Classifiers that differentiated between those with trauma exposure 
(PTSD and TE participants) and those without (HC participants) indicated that exposure was associated with poorer subjective and objective sleep quality (that is, a higher PSQI score and less SWS percentage), elevated evening adrenaline, and poorer neutral declarative memory at post-sleep awakening (Figure 1 A-C). These findings are important because previous research has shown that activation of the sympathetic branch of the autonomic nervous system in the evening - proposed in this study by descriptively elevated adrenaline in trauma-exposed individuals - is associated with poor sleep quality, including disrupted early-night SWS (Mitchell \& Weinshenker, 2010). Because SWS is critical for memory consolidation (Feld \& Diekelmann, 2015; Marshall, Helgadottir, Molle, \& Born, 2006; Walker, 2009), the implication here is that lowered SWS percentage in trauma-exposed individuals may contribute to poor neutral declarative memory, a conjecture consistent with data reported in one other study (van Liempt et al., 2011).

This conjecture is supported, in part, by the observation that poorer neutral declarative memory performance was associated with trauma exposure only at post-sleep awakening, rather than before sleep or across waking according to the SVM model. This interpretation is tempered somewhat because the reported measure of neutral declarative memory did not control for pre-sleep learning. Between-group comparisons of several measures of neutral 
Role of Sleep, Memory and Adrenergic Metabolites in PTSD

declarative memory performance also demonstrated post-sleep patterns of deficits in trauma-exposed individuals. Collectively, these findings permit the cautious speculation that sleep-dependent memory consolidation is impaired in individuals with trauma exposure. We are currently analyzing data from a study designed to test that speculation.

Interestingly, this cluster of variables only differentiated traumaexposed individuals from HC participants - it did not distinguish those with trauma exposure but no PTSD from those with PTSD. One possible explanation is that although poor sleep quality (measured both subjectively and objectively), elevated evening adrenaline and poor neutral declarative memory at post-sleep awakening are all associated with trauma, sleep fragmentation is a core feature distinguishing trauma-exposed individuals with and without PTSD. An alternative explanation with respect to sexual assault survivors, who comprised the entirety of this sample, is that trauma-exposed individuals without PTSD still bear a high load of trauma symptomology that makes them more similar to their counterparts with PTSD than to HC participants. Support for this speculation emerges from a meta-analysis of neutral declarative memory deficits in PTSD which found that PTSD-diagnosed participants had poorer memory in comparison with TE participants, except for survivors of sexual assault, where there were no such between-group differences (Johnsen \& Asbjornsen, 2008). 


\subsection{Strengths and limitations}

A strength of the study design is that it controlled for a series of variables (time since trauma, type of trauma, childhood trauma exposure, and a history of alcohol or substance abuse) that might otherwise have offset results and interpretation of the main findings. Such confounds have been identified across the literature, and constitute a significant barrier towards identifying reproducible objective markers of sleep and memory in PTSD. Additionally, most studies investigating the underlying neurobiology and disturbances of circadian rhythm in PTSD have been conducted using male combat veterans; few PTSD studies focus on women. Because women are more likely to develop PTSD (Christiansen \& Hansen, 2015), this highlights an important aspect of the design. Moreover, although many studies investigating sleep and memory deficits in PTSD have included trauma survivors, diagnosed with or without PTSD, they often do not also incorporate a trauma-free healthy control group. Finally, studies of the application of bio-statistical tools for integrating and cross-validating such measures for predicting PTSD diagnosis are scarce. Advantages of the SVM technique over conventional linear regression include: (a) the quality of out-ofsample generalisation and ease of training; $(b)$ the use of maximummargin hyper-planes in order to maximise the SVMs ability to predict the correct classification of previously unseen samples; $(c)$ the use 
Role of Sleep, Memory and Adrenergic Metabolites in PTSD

of hold-out cross validation, which are capable of achieving superior accuracies than those derived from conventional approaches; and (iv) the use of RFE to identify a unique set of features with maximum predictive value (i.e. classifiers). We anticipate this framework can be extended for future studies collecting a range of clinical, PSG, and/or biological markers from samples of individuals with psychiatric diagnoses.

One limitation is that participants in the PTSD and TE groups had unmatched depression severity. Previous studies show that depression severity increases proportionally with PTSD symptomology in sexual assault survivors (Au, Dickstein, Comer, Salters-Pedneault, \& Litz, 2013). Given this clinical finding, our sample represents the clinical 'norm'. However, we are unable to fully delineate the specific contributions of PTSD and depression symptomology. Furthermore, although our results suggest features that distinguish PTSD, TE, and HC participants, our study does not provide direct evidence of relationships, causal or otherwise, among these measures. Moreover, the current work characterises these data with the use of a priori defined clinical diagnoses, which may potentially limit prediction/classification accuracies. Finally, this exploratory study applies leave-one-out cross validation in a moderately sized cohort, thus necessitating split sample crossvalidation in a larger, non-overlapping sample of participants. 


\subsection{Conclusion}

Using machine learning techniques, we were able to differentiate between individuals with (a) trauma exposure versus no trauma exposure or psychopathology, and (b) those with a PTSD diagnosis versus those with trauma exposure but without PTSD. The analyses yielded moderate-to-high classification accuracies. With respect to (a), the findings showed that sleep variables (measured both subjectively and objectively), evening metadrenaline metabolites, and neutral declarative memory performance after a period of sleep differentiated between those with trauma exposure and those without. With respect to (b), the findings showed that only sleep variables differentiated those with a PTSD diagnosis from those with trauma exposure but without PTSD. Our findings lend support to the hypothesis that sleep disruption is a core feature of PTSD, and provide a framework for future sleep- and memory-based investigations into the pathophysiology of the disorder. Specifically, we hope to inspire future, larger sleep- and memory-based research to apply multivariate classification techniques to model PTSD diagnosis. Regarding clinical implications of the present findings, understanding sleep disruption as a central component of PTSD, and consequently testing whether targeted sleep interventions alleviate other symptoms of the disorder, may pave the way for novel treatment approaches. 
Role of Sleep, Memory and Adrenergic Metabolites in PTSD

\section{Acknowledgements}

This work was supported by the A.W. Mellon Foundation and the National Research Foundation. These funders provided individual financial support to GL - they had no role in the study design; collection, analysis and interpretation of the data; writing of the report; or in the decision to submit the article for publication. 
Role of Sleep, Memory and Adrenergic Metabolites in PTSD

\title{
References
}

Agorastos, A., Kellner, M., Baker, D. G., \& Otte, C. (2014).

When time stands still: an integrative review on the role of chronodisruption in posttraumatic stress disorder. Current Opinion in Psychiatry, 27(5), 385-392.

doi:10.1097/YCO.0000000000000079

\author{
Au, T. M., Dickstein, B. D., Comer, J. S., Salters-Pedneault, K., \\ \& Litz, B. T. (2013). Co-occurring posttraumatic stress and \\ depression symptoms after sexual assault: A latent profile \\ analysis. Journal of Affective Disorders, 149(1), 209-216. \\ doi:10.1016/j.jad.2013.01.026
}

Beck, A. T., Steer, R. A., \& Brown, G. K. (1996). Manual for the Beck Depression Inventory-II. San Antonio, TX: Psychological Corporation.

Blake, D. D., Weathers, W., Nagy, L. M., Kaloupek, D. G., Gusman, F. D., Charney, D. S., \& Keane, T. M. (1995). The development of a clinician-administered PTSD scale. Journal of Traumatic Stress, 8, 75-90.

Bremner, J. D., Randall, P., Scott, T. M., Bronen, R. A., Seibyl, J. P., Southwick, S. M., . . . Innis, R. B. (1995). MRI-based measurement of hippocampal volume in patients with combat- 
Role of Sleep, Memory and Adrenergic Metabolites in PTSD

related posttraumatic stress disorder. American Journal of Psychiatry, 152(7), 973-981.

Brewin, C. R., Kleiner, J. S., Vasterling, J. J., \& Field, A. P. (2007). Memory for emotionally neutral information in posttraumatic stress disorder: A meta-analytic investigation. Journal of Abnormal Psychology, 116(3), 448-463.

doi:10.1037/0021-843X.116.3.448

Burtis, C. A., Ashwood, E. R., \& Bruns, D. E. (2012). Tietz Textbook of Clinical and Molecular Diagnostics. USA: Elsevier.

Buysse, D. J., Hall, M. L., Strollo, P. J., Kamarck, T. W., Owens, J., Lee, L., . . Matthews, K. A. (2008). Relationships between the Pittsburgh Sleep Quality Index (PSQI), Epworth Sleepiness Scale (ESS), and clinical/polysomnographic measures in a community sample. Journal of Clinical Sleep Medicine, 4(6), $563-571$.

Buysse, D. J., Reynolds, C. F., 3rd, Monk, T. H., Berman, S. R., \& Kupfer, D. J. (1989). The Pittsburgh Sleep Quality Index: a new instrument for psychiatric practice and research. Psychiatry Research, 28(2), 193-213. 
Role of Sleep, Memory and Adrenergic Metabolites in PTSD

Christiansen, D. M., \& Hansen, M. (2015). Accounting for sex differences in PTSD: A multi-variable mediation model. Eur $\mathrm{J}$ Psychotraumatol, 6, 26068. doi:10.3402/ejpt.v6.26068

Davis, J. L., \& Wright, D. C. (2007). Randomized clinical trial for treatment of chronic nightmares in trauma-exposed adults. Journal of Traumatic Stress, 20(2), 123-133.

doi:10.1002/jts.20199

Dow, B. M., Kelsoe, J. R., Jr., \& Gillin, J. C. (1996). Sleep and dreams in Vietnam PTSD and depression. Biological Psychiatry, 39(1), 42-50. doi:0006-3223(95)00103-4 [pii] 10.1016/00063223(95)00103-4

Engdahl, B. E., Eberly, R. E., Hurwitz, T. D., Mahowald, M. W., \& Blake, J. (2000). Sleep in a community sample of elderly war veterans with and without posttraumatic stress disorder. Biological Psychiatry, 47(6), 520-525. doi:S00063223(99)00201-2 [pii]

Feld, G. B., \& Diekelmann, S. (2015). Sleep smart-optimizing sleep for declarative learning and memory. Front Psychol, 6, 622. doi:10.3389/fpsyg.2015.00622 
Role of Sleep, Memory and Adrenergic Metabolites in PTSD

Fuller, K. H., Waters, W. F., \& Scott, O. (1994). An investigation

of slow-wave sleep processes in chronic PTSD patients. Journal of Anxiety Disorders, 8, 227-236.

Galatzer-Levy, I. R., Karstoft, K. I., Statnikov, A., \& Shalev, A. Y. (2014). Quantitative forecasting of PTSD from early trauma responses: a Machine Learning application. Journal of Psychiatry Research, 59, 68-76. doi:10.1016/j.jpsychires.2014.08.017

Germain, A. (2013). Sleep disturbances as the hallmark of PTSD: where are we now? American Journal of Psychiatry, 170(4), 372-382. doi:10.1176/appi.ajp.2012.12040432

Germain, A., Richardson, R., Moul, D. E., Mammen, O., Haas, G., Forman, S. D., . . Nofzinger, E. A. (2012). Placebocontrolled comparison of prazosin and cognitive-behavioral treatments for sleep disturbances in US Military Veterans. Journal of Psychosomatic Research, 72(2), 89-96. doi:10.1016/j.jpsychores.2011.11.010

Giosan, C., Malta, L. S., Wyka, K., Jayasinghe, N., Evans, S., Difede, J., \& Avram, E. (2015). Sleep disturbance, disability, and posttraumatic stress disorder in utility workers. J Clin Psychol, 71(1), 72-84. doi:10.1002/jclp.22116 
Role of Sleep, Memory and Adrenergic Metabolites in PTSD

Glaubman, H. M., Miculincer, M., Porat, A., Wasserman, O., \&

Birger, M. (1990). Sleep of chronic posttraumatic patients.

Journal of Traumatic Stress, 3, 255-256.

Goerke, M., Muller, N. G., \& Cohrs, S. (2017). Sleep-dependent memory consolidation and its implications for psychiatry. Journal of Neural Transmission (Vienna), 124(Suppl 1), 163-178.

doi:10.1007/s00702-015-1476-3

Habukawa, M., Uchimura, N., Maeda, M., Kotorii, N., \& Maeda, H. (2007). Sleep findings in young adult patients with posttraumatic stress disorder. Biological Psychiatry, 62(10), 1179-1182. doi:10.1016/j.biopsych.2007.01.007

Hefez, A., Metz, L., \& Lavie, P. (1987). Long-term effects of extreme situational stress on sleep and dreaming. American Journal of Psychiatry, 144(3), 344-347.

Hurwitz, T. D., Mahowald, M. W., Kuskowski, M., \& Engdahl, B. E. (1998). Polysomnographic sleep is not clinically impaired in Vietnam combat veterans with chronic posttraumatic stress disorder. Biological Psychiatry, 44(10), 1066-1073. doi:S00063223(98)00089-4 [pii]

Johnsen, G. E., \& Asbjornsen, A. E. (2008). Consistent impaired verbal memory in PTSD: a meta-analysis. Journal of Affective 
Role of Sleep, Memory and Adrenergic Metabolites in PTSD

Disorders, 111(1), 74-82. doi:S0165-0327(08)00089-X [pii]

10.1016/j.jad.2008.02.007

Kobayashi, I., Boarts, J. M., \& Delahanty, D. L. (2007).

Polysomnographically measured sleep abnormalities in PTSD: a meta-analytic review. Psychophysiology, 44(4), 660-669.

doi:PSYP537 [pii] 10.1111/j.1469-8986.2007.537.x

Kobayashi, I., Huntley, E., Lavela, J., \& Mellman, T. A. (2012).

Subjectively and objectively measured sleep with and without posttraumatic stress disorder and trauma exposure. Sleep, 35(7), 957-965. doi:10.5665/sleep.1960

Kobayashi, I., Lavela, J., \& Mellman, T. A. (2014). Nocturnal autonomic balance and sleep in PTSD and resilience. Journal of Traumatic Stress, 27(6), 712-716. doi:10.1002/jts.21973

Lavie, P., Hefez, A., Halperin, G., \& Enoch, D. (1979). Longterm effects of traumatic war-related events on sleep. American Journal of Psychiatry, 136(2), 175-178.

Lipinska, G., \& Thomas, K. G. F. (2017). Better Sleep in a Strange Bed? Sleep Quality in South African Women with Posttraumatic Stress Disorder. Front Psychol, 8, 1555. doi:10.3389/fpsyg.2017.01555 
Role of Sleep, Memory and Adrenergic Metabolites in PTSD

Lipinska, G., Timol, R., Kaminer, D., \& Thomas, K. G. (2014).

Disrupted rapid eye movement sleep predicts poor declarative memory performance in post-traumatic stress disorder. Journal of Sleep Research, 23(3), 309-317. doi:10.1111/jsr.12122

Lo, J. C., Groeger, J. A., Cheng, G. H., Dijk, D. J., \& Chee, M. W. (2016). Self-reported sleep duration and cognitive performance in older adults: a systematic review and metaanalysis. Sleep Medicine, 17, 87-98. doi:10.1016/j.sleep.2015.08.021

Marshall, L., Helgadottir, H., Molle, M., \& Born, J. (2006). Boosting slow oscillations during sleep potentiates memory. Nature, 444(7119), 610-613. doi:10.1038/nature05278

Mellman, T. A., Bustamante, V., Fins, A. I., Pigeon, W. R., \& Nolan, B. (2002). REM sleep and the early development of posttraumatic stress disorder. American Journal of Psychiatry, 159(10), 1696-1701.

Mellman, T. A., Kobayashi, I., Lavela, J., Wilson, B., \& Hall Brown, T. S. (2014). A relationship between REM sleep measures and the duration of posttraumatic stress disorder in a young adult urban minority population. Sleep, 37(8), 1321-1326. doi:10.5665/sleep.3922 
Role of Sleep, Memory and Adrenergic Metabolites in PTSD

Mellman, T. A., Kulick-Bell, R., Ashlock, L. E., \& Nolan, B.

(1995). Sleep events among veterans with combat-related

posttraumatic stress disorder. American Journal of Psychiatry, 152(1), 110-115.

Mellman, T. A., Kumar, A., Kulick-Bell, R., Kumar, M., \& Nolan, B. (1995). Nocturnal/daytime urine noradrenergic measures and sleep in combat-related PTSD. Biological Psychiatry, 38(3), 174179. doi:0006-3223(94)00238-X [pii] 10.1016/00063223(94)00238-X

Mikulincer, M., Glaubman, H., Wasserman, O., Porat, A., \& Birger, M. (1989). Control-related beliefs and sleep characteristics of posttraumatic stress disorder patients. Psychological Reports, 65(2), 567-576. doi:10.2466/pr0.1989.65.2.567

Mitchell, H. A., \& Weinshenker, D. (2010). Good night and good luck: norepinephrine in sleep pharmacology. Biochemical Pharmacology, 79(6), 801-809. doi:10.1016/j.bcp.2009.10.004

Naccarato, A., Gionfriddo, E., Sindona, G., \& Tagarelli, A. (2014). Development of a simple and rapid solid phase microextraction-gas chromatography-triple quadrupole mass spectrometry method for the analysis of dopamine, serotonin 
Role of Sleep, Memory and Adrenergic Metabolites in PTSD

and norepinephrine in human urine. Analytica chimica $\operatorname{acta}(810), 17-24$.

Pace-Schott, E. F., Germain, A., \& Milad, M. R. (2015). Sleep and REM sleep disturbance in the pathophysiology of PTSD: the role of extinction memory. Biology of Mood and Anxiety Disorders, 5, 3. doi:10.1186/s13587-015-0018-9

Peaston, R. T., \& Weinkove, C. (2004). Measurement of catecholamines and their metabolites. Annals of Clinical Biochemistry, 41, 17-38.

Pienaar, I., Shuttleworth-Edwards, A. B., Klopper, C. C., \& Radloff, S. (2016). Wechsler Adult Intelligence Scale-Fourth Edition preliminary normative guidelines for educationally disadvantaged Xhosa-speaking individuals. South African Journal of Psychology, 47(2), 159-170. doi:10.1177/0081246316654805

Pigeon, W. R., \& Gallegos, A. M. (2015). Posttraumatic Stress Disorder and Sleep. Sleep Medicine Clinics, 10(1), 41-48. doi:10.1016/j.jsmc.2014.11.010

Raskind, M. A., Peterson, K., Williams, T., Hoff, D. J., Hart, K., Holmes, H., . . Peskind, E. R. (2013). A trial of prazosin for 
Role of Sleep, Memory and Adrenergic Metabolites in PTSD

combat trauma PTSD with nightmares in active-duty soldiers returned from Iraq and Afghanistan. American Journal of Psychiatry, 170(9), 1003-1010.

doi:10.1176/appi.ajp.2013.12081133

Rechtschaffen, A., \& Kales, A. (1968). A manual of standardized terminology, techniques and scoring system for sleep stages of human subjects: US Government Printing Office, US Public Health Service.

Ross, R. J., Ball, W. A., Dinges, D. F., Kribbs, N. B., Morrison, A. R., Silver, S. M., \& Mulvaney, F. D. (1994). Motor dysfunction during sleep in posttraumatic stress disorder. Sleep, 17(8), 723732.

Sheehan, D. V., Lecrubier, Y., Sheehan, K. H., Amorim, P., Janavs, J., Weiller, E., . . Dunbar, G. C. (1998). The MiniInternational Neuropsychiatric Interview (M.I.N.I.): The development and validation of a structured diagnostic psychiatric interview for DSM-IV and ICD-10. Journal of Clinical Psychiatry, 59, 22-33.

Sheslow, D., \& Adams, W. (2003). Wide Range Assessment of Memory and Learning Second Edition Administration and 
Role of Sleep, Memory and Adrenergic Metabolites in PTSD

Technical Manual. Lutz, FL: Psychological Assessment Resources.

Simon, R., Lam, A., Li, M.C., Ngan, M., Menenzes, S., \& Zhao, Y. (2007). Analysis of gene expression data using BRBArrayTools. Cancer Informatics, 3, 11-17.

Spoormaker, V. I., \& Montgomery, P. (2008). Disturbed sleep in post-traumatic stress disorder: secondary symptom or core feature? Sleep Medicine Reviews, 12(3), 169-184. doi:10.1016/j.smrv.2007.08.008

Talbot, L. S., Maguen, S., Metzler, T. J., Schmitz, M., McCaslin, S. E., Richards, A., . . Neylan, T. C. (2014). Cognitive behavioral therapy for insomnia in posttraumatic stress disorder: a randomized controlled trial. Sleep, 37(2), 327-341. doi:10.5665/sleep.3408

Torgo, L. (2010). Data Mining using R: learning with case studies. New York: CRC Press.

van Liempt, S., Arends, J., Cluitmans, P. J., Westenberg, H. G., Kahn, R. S., \& Vermetten, E. (2013). Sympathetic activity and hypothalamo-pituitary-adrenal axis activity during sleep in posttraumatic stress disorder: a study assessing polysomnography 
Role of Sleep, Memory and Adrenergic Metabolites in PTSD

with simultaneous blood sampling. Psychoneuroendocrinology, 38(1), 155-165. doi:10.1016/j.psyneuen.2012.05.015

van Liempt, S., Vermetten, E., Lentjes, E., Arends, J., \& Westenberg, H. (2011). Decreased nocturnal growth hormone secretion and sleep fragmentation in combat-related posttraumatic stress disorder; potential predictors of impaired memory consolidation. Psychoneuroendocrinology, 36(9), 13611369. doi:10.1016/j.psyneuen.2011.03.009

Vythilingam, M., Luckenbaugh, D. A., Lam, T., Morgan, C. A., 3rd, Lipschitz, D., Charney, D. S., . . Southwick, S. M. (2005). Smaller head of the hippocampus in Gulf War-related posttraumatic stress disorder. Psychiatry Research, 139(2), 8999. doi:S0925-4927(05)00067-3 [pii] 10.1016/j.pscychresns.2005.04.003

Walker, M. P. (2009). The role of slow wave sleep in memory processing. Journal of Clinical Sleep Medicine, 5(2 Suppl), S2026.

Watkins, C. C., \& Treisman, G. J. (2015). Cognitive impairment in patients with AIDS - prevalence and severity. HIV AIDS (Auckland), 7, 35-47. doi:10.2147/HIV.S39665 
Role of Sleep, Memory and Adrenergic Metabolites in PTSD

Wechsler, D. (1997). Wechsler Memory Scale-Third Edition

(WMS-III) administration and scoring manual. San Antonio: The Psychological Corporation.

Wechsler, D. (1999). Wechsler Abbreviated Scale of Intelligence (WASI). San Antonio: Psychological Corporation.

Werner, K. B., Griffin, M. G., \& Galovski, T. E. (2016). Objective and subjective measurement of sleep disturbance in female trauma survivors with posttraumatic stress disorder. Psychiatry Research, 240, 234-240. doi:10.1016/j.psychres.2016.04.039 Woodward, S. H., Murburg, M. M., \& Bliwise, D. L. (2000). PTSD-related hyperarousal assessed during sleep. Physiology and Behavior, 70(1-2), 197-203.

Yetkin, S., Aydin, H., \& Ozgen, F. (2010). Polysomnography in patients with post-traumatic stress disorder. Psychiatry and Clinical Neuroscience, 64(3), 309-317. doi:PCN2084 [pii] 10.1111/j.1440-1819.2010.02084. 
Table 1. Demographic and clinical characteristics of all participants $(\mathrm{N}=60)$.

\begin{tabular}{|c|c|c|c|c|c|c|}
\hline & $\begin{array}{c}\mathrm{HC} \\
(\mathrm{N}=20)\end{array}$ & $\begin{array}{c}\text { TE } \\
(\mathrm{N}=20)\end{array}$ & $\begin{array}{r}\text { PTSD } \\
(\mathrm{N}=20)\end{array}$ & \multirow[b]{2}{*}{$\begin{array}{l}F / H(d f \\
=2,57)\end{array}$} & \multirow[b]{2}{*}{$P$} & \multirow[b]{2}{*}{ Post-Hoc Significance } \\
\hline & Mean $\pm S D$ & Mean $\pm S D$ & Mean $\pm S D$ & & & \\
\hline Age & $25.30 \pm 4.62$ & $24.50 \pm 4.41$ & $25.50 \pm 4.46$ & 0.793 & 0.673 & n.s. \\
\hline Education (years) & $12.90 \pm 1.99$ & $12.50 \pm 2.04$ & $11.65 \pm 1.63$ & 4.880 & 0.087 & n.s. \\
\hline WASI PIQ ${ }^{\dagger}$ & $83.80 \pm 14.21$ & $83.00 \pm 12.12$ & $78.05 \pm 14.77$ & 2.891 & 0.236 & n.s. \\
\hline MAST & $0.70 \pm 1.12$ & $0.85 \pm 1.39$ & $1.00 \pm 1.02$ & 1.737 & 0.420 & n.s. \\
\hline PSQI & $4.15 \pm 2.65$ & $6.22 \pm 3.25$ & $9.75 \pm 4.11$ & 19.354 & $<0.001$ & PTSD > TE; PTSD > HC; TE > HC \\
\hline BDI-II & $6.20 \pm 3.41$ & $17.89 \pm 7.80$ & $30.60 \pm 6.05$ & 43.130 & $<0.001$ & PTSD > TE; PTSD > HC; TE > HC \\
\hline Normal bedtime & $22 \mathrm{~h} 50 \pm 0.9 \mathrm{~h}$ & $22 \mathrm{~h} 55 \pm 1.0 \mathrm{~h}$ & $21 \mathrm{~h} 10 \pm 1.0 \mathrm{~h}$ & 6.812 & 0.049 & TE > PTSD; HC > PTSD \\
\hline Nicotine: $N(\%)$ & $5(25 \%)$ & $1(5 \%)$ & $6(30 \%)$ & - & - & n.s. \\
\hline Time since trauma & - & $1.26 \pm 1.06$ & $1.26 \pm 1.03$ & 1.800 & 0.892 & n.s. \\
\hline Total counsel sessions & - & $4.20 \pm 3.00$ & $3.94 \pm 1.85$ & 1.039 & 0.308 & n.s. \\
\hline CAPS & - & $29.50 \pm 11.65$ & $68.80 \pm 13.46$ & 29.293 & $<0.001$ & PTSD > TE \\
\hline MINI total & - & $2.05 \pm 1.32$ & $3.65 \pm 1.53$ & 9.270 & 0.002 & PTSD > TE \\
\hline
\end{tabular}

Shapiro-Wilk test was used to assess normality of variables and either a one-way analysis of variance (ANOVA) or Kruskal-Wallis ANOVA with post hoc Tukey correction was implemented accordingly. Abbreviations: WASI PIQ, Wechsler Abbreviated Scale of Intelligence Performance Intelligence Quotient; MAST, Michigan Alcohol Screening Test; PSQI, Pittsburgh Sleep Quality Index; BDI-II, Beck Depression Inventory-Second Edition; Nicotine, number of smoking participants; Time since trauma, given in years; CAPS, Clinician-Administered PTSD Scale; MINI, Mini-International Neuropsychiatric Interview; MINI total, number of MINI diagnoses; n.s., not significant.

${ }^{\dagger} \mathrm{Q}$ scores are in the average range for non-first language English speaking South African adults with 12 years of poor quality education (Pienaar, Shuttleworth-Edwards, Klopper, \& Radloff, 2016) 
Role of Sleep, Memory and Adrenergic Metabolites in PTSD

Table 2. Correlations between PTSD severity (CAPS Total) and subjective sleep (PSQI) with clinical measurements across PTSD and TE groups $(\mathrm{N}=40)$.

\begin{tabular}{|c|c|c|c|c|c|c|}
\hline & \multicolumn{2}{|c|}{ PTSD and TE $(n=40)$} & \multicolumn{2}{|c|}{ PTSD $(n=20)$} & \multicolumn{2}{|c|}{$\operatorname{TE}(n=20)$} \\
\hline & $\begin{array}{c}\text { PSQI } \\
\text { R-value } \\
(P \text {-value }) \\
\end{array}$ & $\begin{array}{l}\text { CAPS Total } \\
\text { R-value } \\
(P \text {-value })\end{array}$ & $\begin{array}{c}\text { PSQI } \\
\text { R-value } \\
(P \text {-value }) \\
\end{array}$ & $\begin{array}{l}\text { CAPS Total } \\
\text { R-value } \\
(P \text {-value }) \\
\end{array}$ & $\begin{array}{c}\text { PSQI } \\
\text { R-value ( } P \text { - } \\
\text { value) } \\
\end{array}$ & $\begin{array}{l}\text { CAPS Total } \\
\text { R-value } \\
(P \text {-value }) \\
\end{array}$ \\
\hline Time since trauma & $0.230(0.150)$ & $0.063(0.700)$ & $0.39(0.089)$ & $0.293(0.21)$ & $-0.037(0.876)$ & $-0.199(0.400)$ \\
\hline BDI & $0.553(<0.001)^{*}$ & $0.804(<0.001)^{*}$ & $0.501(0.024)^{*}$ & $0.542(0.013)^{*}$ & $0.436(0.055)$ & $0.584(<0.01)^{*}$ \\
\hline PSQI & - & $0.654(<0.001)^{*}$ & - & $0.588(<0.01)^{*}$ & - & $0.654(<0.01)^{*}$ \\
\hline CAPS Total & $0.654(<0.001)^{*}$ & - & $0.588(<0.01)^{*}$ & - & $0.654(<0.01)^{*}$ & - \\
\hline CAPS B & $0.550(<0.001)^{*}$ & $0.827(<0.001)^{*}$ & $0.465(0.039)^{*}$ & $0.762(<0.001)^{*}$ & $0.258(0.271)$ & $0.462(0.040)^{*}$ \\
\hline CAPS C & $0.581(<0.001)^{*}$ & $0.900(<0.001)^{*}$ & $0.462(0.040)^{*}$ & $0.79(<0.001)^{*}$ & $0.463(0.040)^{*}$ & $0.698(<0.001)^{*}$ \\
\hline CAPS D & $0.576(<0.001)^{*}$ & $0.816(<0.001)^{*}$ & $0.304(0.193)$ & $0.533(0.015)^{*}$ & $0.511(0.021)^{*}$ & $0.751(<0.001)^{*}$ \\
\hline MINI Total & $0.381(0.015)^{*}$ & $0.604(<0.001)^{*}$ & $-0.059(0.805)$ & $0.201(0.396)$ & $0.632(<0.01)^{*}$ & $0.839(<0.001)^{*}$ \\
\hline MINI depression & $0.379(0.016)^{*}$ & $0.496(<0.01)^{*}$ & $-0.199(0.401)$ & $-0.06(0.802)$ & $0.665(<0.01)^{*}$ & $0.872(<0.001)^{*}$ \\
\hline MINI suicidality & $0.128(0.430)$ & $0.098(0.550)$ & $0.072(0.762)$ & $-0.015(0.949)$ & $0.172(0.468)$ & $0.314(0.177)$ \\
\hline MINI panic disorder & $-0.017(0.923)$ & $0.073(0.661)$ & $-0.15(0.527)$ & $-0.157(0.510)$ & -0.009 (0.969) & $-0.253(0.281)$ \\
\hline MINI agoraphobia & $-0.066(0.694)$ & $0.325(0.041)^{*}$ & $-0.229(0.332)$ & $0.184(0.439)$ & $-0.183(0.441)$ & $0.117(0.623)$ \\
\hline MINI PTSD & $0.550(<0.001)^{*}$ & $0.655(<0.001)^{*}$ & $0.079(0.740)$ & $0.154(0.517)$ & $0.665(<0.01)^{*}$ & $0.872(<0.001)^{*}$ \\
\hline MINI hypomanic episode & $0.359(0.023)^{*}$ & $0.298(0.062)$ & $0.458(0.042)^{*}$ & $0.234(0.321)$ & $N A$ & $N A$ \\
\hline MINI dysthymia & $0.130(0.421)$ & $0.222(0.174)$ & $-0.145(0.541)$ & $-0.098(0.681)$ & $0.293(0.210)$ & $0.176(0.457)$ \\
\hline MINI OCD & $0.244(0.130)$ & $0.179(0.270)$ & $0.315(0.176)$ & $0.336(0.148)$ & $0.206(0.384)$ & $0.273(0.244)$ \\
\hline MINI social $p$ & $0.145(0.373)$ & $0.318(0.045)^{*}$ & $0.012(0.959)$ & $0.386(0.093)$ & $N A$ & $N A$ \\
\hline MINI mood disorders & $-0.014(0.934)$ & $0.257(0.113)$ & $-0.135(0.569)$ & $0.301(0.198)$ & $N A$ & $N A$ \\
\hline
\end{tabular}

Spearman's correlation was used to assess relationships of PSQI and CAPS to clinical diagnostic criteria. Abbreviations: BDI, Beck Depression Inventory; PSQI, Pittsburgh Sleep Quality Index; CAPS, Clinician Administered PTSD Scale; MINI, Mini-International Neuropsychiatric Interview. $P$-value $<0.05\left(^{*}\right)$ is considered significant. 

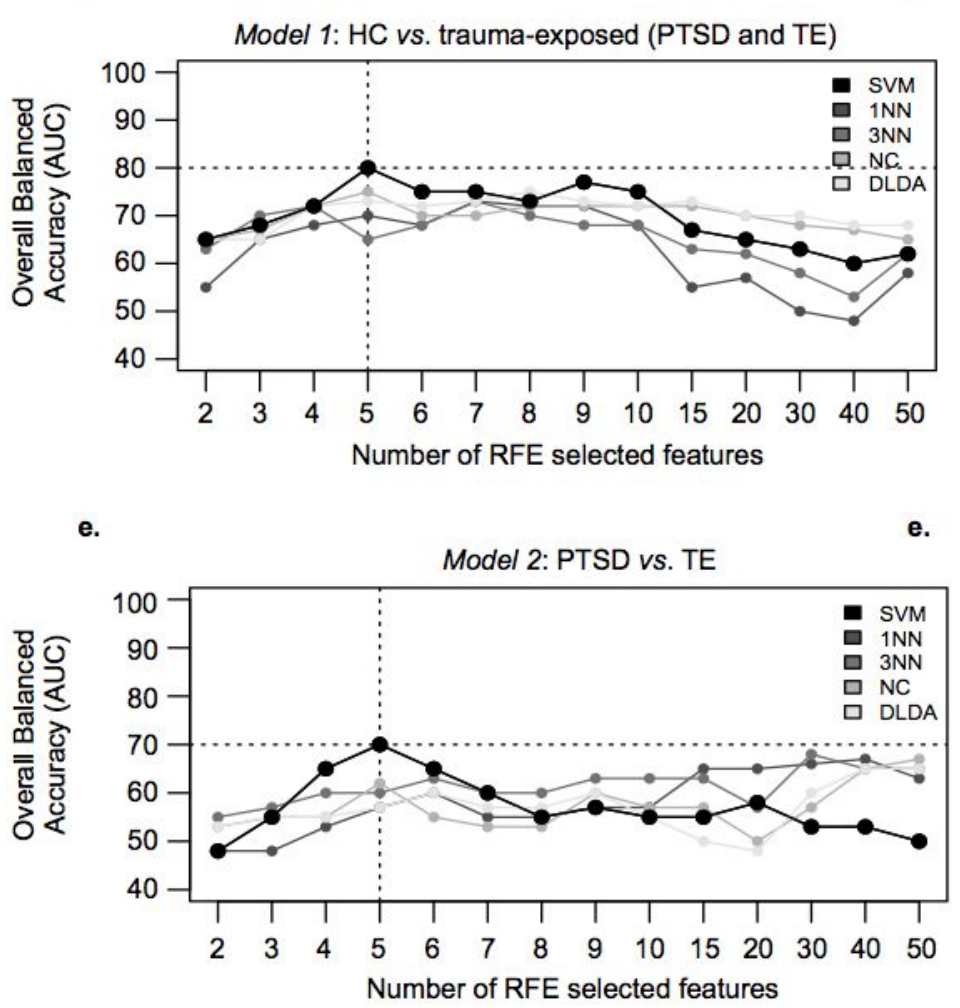

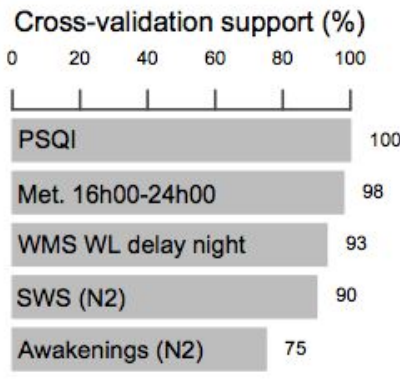

c.

\begin{tabular}{|l|ccccc|}
\hline \multicolumn{7}{|c|}{ Model 1: Top 5 Features } \\
\hline Sensitivity & NC & 1NN & 3NN & DLDA & SVM \\
\hline Specificity & 0.75 & 0.78 & 0.80 & 0.75 & 0.87 \\
AUC & 0.65 & 0.45 & 0.43 & 0.67 & 0.65 \\
$95 \%$ C.I. & 0.72 & 0.68 & 0.68 & 0.72 & 0.80 \\
\hline
\end{tabular}

f.

Cross-validation support (\%)

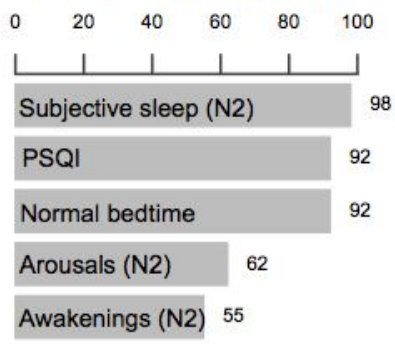

Model 2: Top 5 Features

\begin{tabular}{|l|ccccc|}
\hline & NC & 1NN & 3NN & DLDA & SVM \\
\hline Sensitivity & 0.76 & 0.70 & 0.72 & 0.75 & 0.80 \\
Specificity & 0.33 & 0.36 & 0.38 & 0.39 & 0.61 \\
AUC & 0.55 & 0.60 & 0.63 & 0.60 & 0.70 \\
$95 \%$ C.I. & \multicolumn{7}{l}{$0.49-0.60$} & $0.48-0.66$ & $500 .-0.68$ & $0.53-0.67$ & $0.66-0.74$ \\
\hline
\end{tabular}


Figure 1. Supervised machine-learning integrating clinical, sleep, memory, and biological data to predict group outcomes. Panel (a): Classifier classifier accuracies achieved when discriminating HC from PTSD and TE groups; and-Panel (b): cross-validation support (i.e., the percentage of times a feature was used in the predictor for a leave-one-out crossvalidation procedure); and-Panel (c): results of the top performing model, which containing-contains 5 features-are displayed; Panel -(d): Classifier classifier accuracies achieved when discriminating PTSD participants from TE participants; Panel and-(e): cross-validation support; Panel and-(f): results from the top top-performing model-containing 5 features are displayed. Gross-validation support is the percentage of times when a feature was used in the predictor for a leave-one-out cross-validation procedure. In each case ${ }_{2}$ supervised class prediction was performed using different combinations of genes withusing Recursive Feature Elimination (RFE) and evaluated with four different multivariate classification methods. For Panels (c) and (f), Sensitivity sensitivity is defined as-the rate of true positives over the rate of true positives plus false negatives, and. Sspecificity is defined as-the rate of true negatives over the rate of true negatives plus false positives. Abbreviations: AUC, overall balanced accuracy; C.I., confidence interval; NC, nearest centroid; $1 \mathrm{NN}$, nearest neighbour; 3NN, three-nearest neighbours; SVM, support vector machines; DLDA, diagonal linear discriminate analysis. 
Supplementary Figure 1. Schematic of the implemented machine-learning and cross-validation pipeline. Leave-one-out cross-validation is initiated by randomly sub-setting one sample (test) and the remaining samples (training set) are subjected to recursive feature elimination feature selection (FS) and internal nested 10-fold cross-validation performed 10 times. The final classifier $(\mathrm{C})$ is applied to predict classification for the original left-out sample. This process is iterated until all samples are left out and a contingency table is populated for each prediction, entailing true positives (TP), true negatives (TN), false positives (FP) and false negatives (FN). These values are used to compute measures of sensitivity, specificity and overall balanced accuracy (AUC). 